\title{
Synchrotron XRD Evaluation of Residual Stresses Introduced by Laser Shock Peening for Steam Turbine Blade Applications
}

\author{
M. Newby ${ }^{1, a^{*}}$, A. Steuwer ${ }^{2, b}$, D. Glaser ${ }^{3,4, c}$, C. Polese ${ }^{4, d}$, \\ D.G. Hattingh ${ }^{5, e}$ and C. Gorny ${ }^{6, f}$ \\ ${ }^{1}$ Eskom Holdings SOC Ltd, Rosherville, Johannesburg, South Africa \\ ${ }^{2}$ University of Malta, Msida MSD 2080, Malta \\ ${ }^{3}$ CSIR National Laser Centre, Brummeria, Pretoria, South Africa \\ ${ }^{4}$ University of the Witwatersrand, Johannesburg, South Africa \\ ${ }^{5}$ Nelson Mandela University, Port Elizabeth, South Africa \\ ${ }^{6}$ Laboratoire PIMM (ENSAM, CNRS, CNAM, Hesam Université), Paris, France \\ amark.newby@eskom.co.za, baxel.steuwer@gmail.com, cdglaser@csir.co.za, \\ dclaudia.polese@wits.ac.za, ${ }^{\mathrm{e}}$ danie.hattingh@mandela.ac.za, ${ }^{\mathrm{f}}$ cyril.gorny@ensam.eu
}

\section{Keywords: Laser Shock Peening, Residual Stress, Synchrotron, Diffraction}

\begin{abstract}
Steam turbines used in the power generation industry are subject to fatigue during normal operation which includes transient events such as start-ups and steady state operation. Surface treatment methods, such as shot peening (SP) and roller burnishing, to induce surface compressive residual stresses in critical areas and improve fatigue life are commonly used, but the depth of the induced residual stresses is limited by the process. Laser shock peening (LSP) is a more recent development that has been applied in the aerospace industry on titanium blades, but is not yet commonly used in the power generation industry. The current research is focused on optimizing LSP parameters for the application of the process on $12 \mathrm{Cr}$ steels used for turbine blades. Evaluation of the induced residual stress was done with both conventional laboratory Xray diffraction (XRD) and synchrotron X-ray diffraction (SXRD) techniques.
\end{abstract}

\section{Introduction}

Thermal energy is the most commonly used source for electricity generation globally, which is often extracted by the use of large steam turbines. The corrosion resistant steel blades of a typical low pressure (LP) rotor operate in a wet steam environment, whilst rotating at speeds in the range of 3000 to $3600 \mathrm{rpm}$. The approximately $1 \mathrm{~m}$ long LP blades therefore see high centrifugal loading, which presents challenges of stress corrosion cracking or corrosion fatigue [1]. The highly stressed fir-tree attachment root, as illustrated in Fig. 2, is conventionally shot peened (SP) in order to introduce beneficial compressive residual stresses to mitigate crack initiation. A catastrophic failure of one of these blades at a South African power station in 2003 resulted in over $€ 100$ million damage, and raised concerns to the effectiveness of the conventional SP treatment for the achievement of uniform coverage over the complex geometry of the fir tree section.

Laser shock peening (LSP) has been identified as an attractive technology for this application in order to potentially enhance the lifespan of the critical LP turbine blades. One of the benefits of a laser-based technology is the precise control of laser parameters, and hence the potential to introduce a beneficial compressive stress field to the desired level. Furthermore, the plasma 
generated on the material surface produces a pressure normal to that surface which is potentially highly attractive to apply a uniform treatment to a complex 3D surface, such as a turbine blade fir-tree. The CSIR National Laser Centre (NLC) and Eskom are currently conducting research into the application of LSP for turbine blade refurbishment in close collaboration with SA universities.

The mechanism of the LSP process is depicted in the schematic in Fig.1. A high intensity laser pulse (duration in the order of nano-seconds) irradiates a metal target to rapidly ionize the surface into a plasma. When the plasma is confined with a medium transparent to the laser pulse, Giga Pascal (GPa) magnitude pressures are generated over a nano-second time frame which drives a shock wave through the metallic target. Plasticity is produced through the surface to a depth whereby the shockwaves no longer exceed the dynamic yield strength of the material. The material's elastic response to the plastic strains is the generation of a beneficial compressive residual stress to depths typically around $1 \mathrm{~mm}$ or greater. A sacrificial protective coating may be applied temporarily during LSP processing in order to prevent a direct laser-material interaction, which ensures a purely mechanical cold-working process. After each LSP application the tape is removed and replaced, thus these increments are generally referred to as tape layers. An alternative technique is to laser shock peen the surface without a protective coating (LSPwC), through a thin water containment film. An initial study conducted on $12 \mathrm{Cr}$ samples at the NLC yielded encouraging results for both LSP and LSPwC processes, however optical analysis of the spatial intensity profile across the laser spot showed significant variation from the desired top-hat profile, illustrated in the right hand images of Fig. 1. As a result a new batch of samples was processed for the results presented in this paper. These samples were treated at Laboratoire PIMM, in Paris.

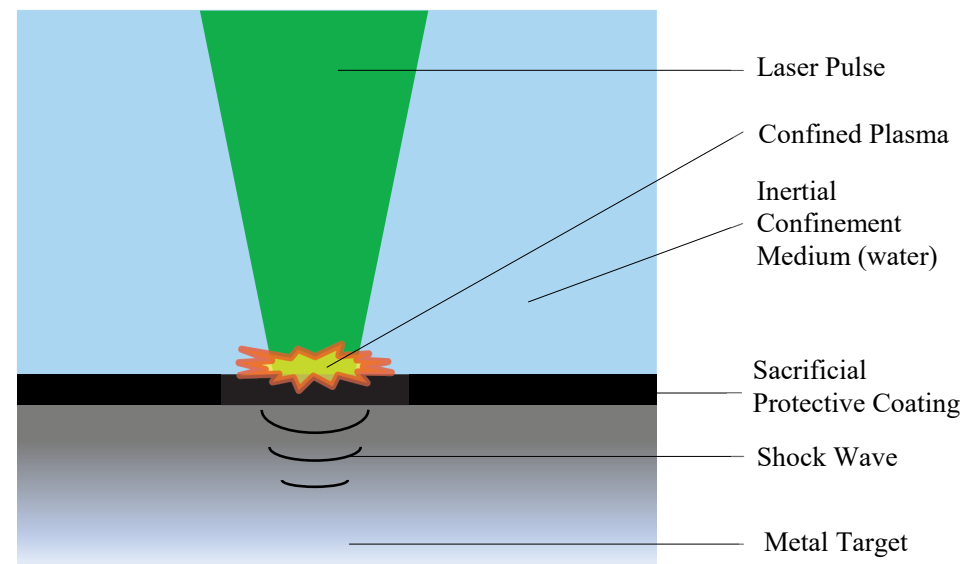

Laser schematic

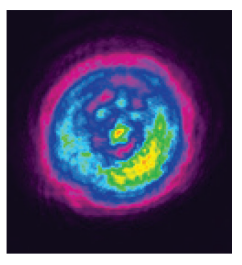

Uneven beam profile

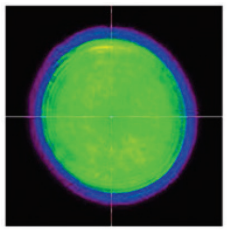

Uniform (top hat) beam profile

Fig. 1: A schematic of the LSP process (left) and of two laser beam profiles (right).

\section{Methodology}

Sample Generation: Samples were extracted from an ex-service turbine blade by removing slices from the fir-tree attachment region as depicted in Fig. 2. A stress relieving cycle of $660^{\circ} \mathrm{C}$ for 20 minutes was performed on the coupons. The samples were wire EDM cut to dimensions of $20 \mathrm{x}$ $20 \times 15 \mathrm{~mm}^{3}$ which were also surface ground. Electro-polishing was applied to remove the surface grinding effects, and hardness was checked to ensure that the stress relieving did not alter the mechanical properties. Laboratory XRD measurements were performed on each sample before and after LSP processing for repeatability verification of sample preparation and LSP processing. 


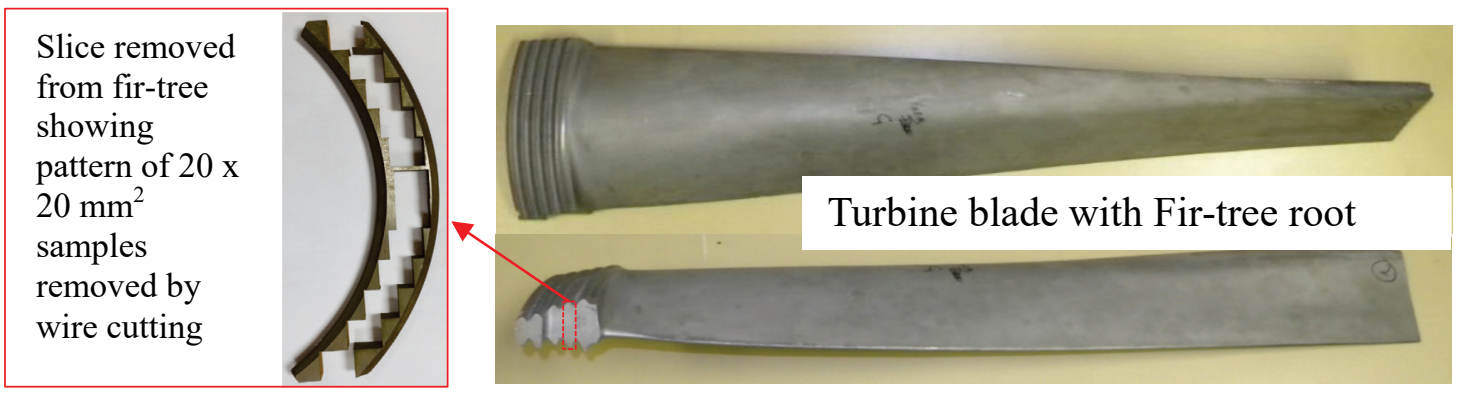

Fig. 2: Turbine blade configuration.

LSP and LSPwC Processing: The application of laser peening was performed at the PIMM Laboratory (ENSAM-CNRS-CNAM) using a Thales GAIA laser system operating at $532 \mathrm{~nm}$ with the sample immersed in a water tank. For the LSP work, a sacrificial thermo-protective overlay in the form of a black PVC tape was used (around $100 \mu \mathrm{m}$ thick with a $30 \mu \mathrm{m}$ adhesive). In order to ensure coating integrity, the spot-to-spot overlap was kept low at around $21.5 \%$. A spot size of $2.0 \mathrm{~mm}$ was selected, as this is approximately the largest diameter spot that would be practical to process within the turbine blade fir-tree as depicted in Fig. 2. A preliminary phase was first conducted in order to determine an appropriate power intensity whereby a constant spot size and overlap were used with varying power intensity from 1 to $8 \mathrm{GW} / \mathrm{cm}^{2}$. Surface XRD measurements were made to identify $5 \mathrm{GW} / \mathrm{cm}^{2}$ as a conservative power intensity operating below the saturation limit of the process due to dielectric breakdown before the target. The LSP processing was performed on the $20 \times 20 \mathrm{~mm}^{2}$ sample face with a $10 \times 10$ $\mathrm{mm}^{2}$ LSP patch as depicted in Fig. 3. The LSPwC processing used two different spot sizes, 0.6 and $0.8 \mathrm{~mm}$ with coverage parameters of $\mathrm{Np}=16.55$ and 33.75, $(\mathrm{Np}=$ the number of pulses per $\mathrm{mm}^{2}$ ).

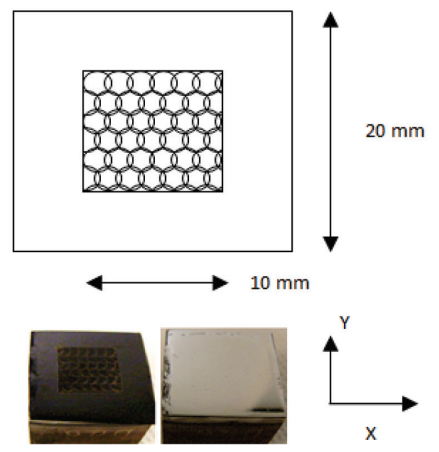

Fig. 3: LSP application pattern.

Laboratory X-ray diffraction: The surface residual stress analysis was performed using XRD measurements with a Proto iXRD instrument from Proto Manufacturing Inc., USA. A Cr-Ka Xray source with a wavelength of $2.291 \AA$ was used with a round $1.0,0.5$ or $0.2 \mathrm{~mm}$ aperture. Reflections from the (211) peak for the steel were used with a goniometer range of either $\pm 30^{\circ}$ or $\pm 25^{\circ}$ with a $3^{\circ}$ oscillation, where the Bragg angle was $156.31^{\circ}$. The measurements were performed as per the $\sin 2 \psi$ technique with a minimum of 7 angles per strain measurement for the surface maps, for the depth profiles 11 angles per measurement were used to improve accuracy. Tri-axial measurements were performed at 0,45 , and 90 degrees in order to obtain the principal stresses. Stresses were calculated using the X-ray elastic constants $-\mathrm{S} 1=1.15 \times 10^{-6} \mathrm{MPa}^{-1}$ and $1 / 2 \mathrm{~S} 2=5.247 \times 10^{-6} \mathrm{MPa}^{-1}$, determined through four-point bend tests.

Synchrotron X-ray diffraction: SXRD measurements were conducted at the ID15A beamline (experiment ME1440) at the ESRF facility in Grenoble, France. Energy dispersive measurements were performed with up to $300 \mathrm{keV}$ energies which allowed for transmission through the $20 \mathrm{~mm}$ dimensions of the samples in order to provide strain measurements in the Y-direction as indicated in the schematic in Fig. 3. The beam dimensions were set to $50 \mu \mathrm{m}$ by $100 \mu \mathrm{m}$, where the smaller dimension is in the depth (i.e. perpendicular to the LSP surface). A diffracting angle of around $3^{\circ}$ results in gauge volume elongation to around $1.9 \mathrm{~mm}$. A measurement time of two minutes was used for each position in the sample. The data was processed using GSAS Pawley 
analysis in order to determine the lattice parameter accounting for multiple peaks for the bcc material. The strains were computed to stresses by assuming a bi-axial stress where an elastic modulus of $204 \mathrm{GPa}$ and Poisson's ratio of 0.3 were used for computation. A small pillar of the material $\left(2 \times 2 \mathrm{~mm}^{2}\right.$ by $10 \mathrm{~mm}$ length) was used in order to determine the stress-free lattice parameter, $\mathrm{d}_{\mathrm{zero}}$.

\section{Results and Discussion}

Fig. 4 shows the XRD surface and SXRD through thickness residual stress results for three different power intensities with one layer of tape. Previous work [2,3] has shown that the depth profiles become more consistent and deeper with the second tape layer and this is reflected in the data variation of up to $100 \mathrm{MPa}$ peak to peak (pk-pk) shown in the plots. There is good correlation between the XRD and SXRD data at the surface of the samples, and a clear indication of the improved depth profile with increasing power intensity.

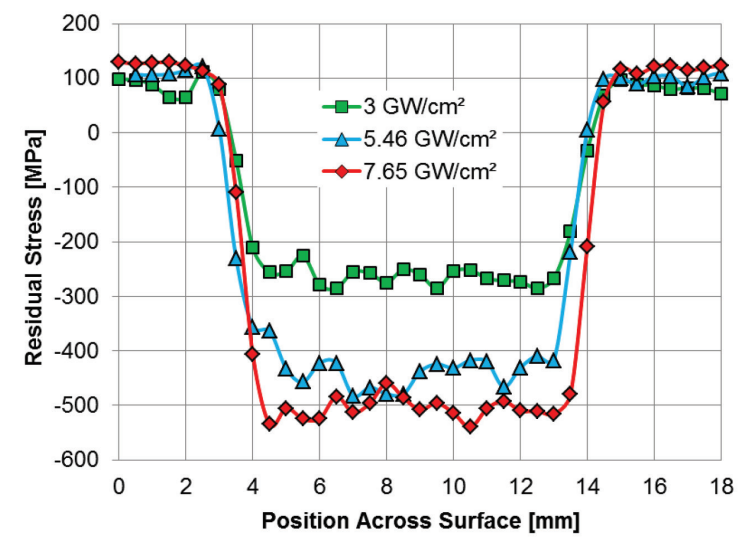

XRD surface residual stress line scans, $1 \mathrm{~mm}$ aperture, $0.5 \mathrm{~mm}$ spatial resolution

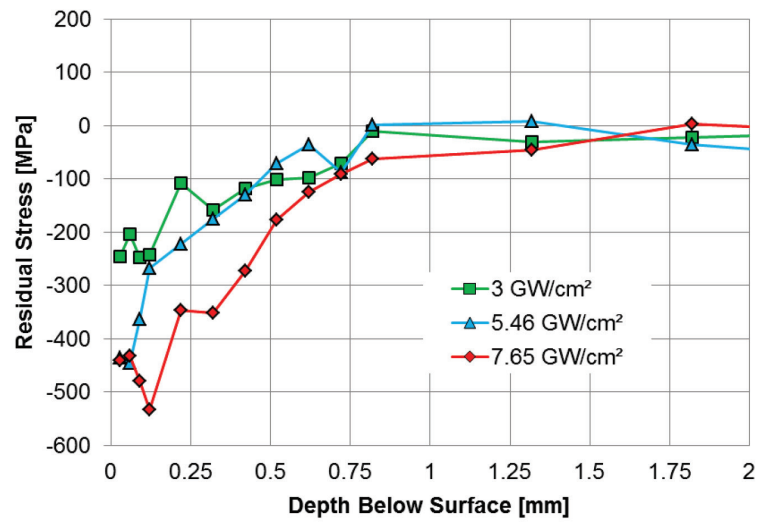

SXRD residual stress depth profiles

Fig. 4: Residual stresses comparisons as function of laser power intensity employed with the LSP processing using one tape layer.

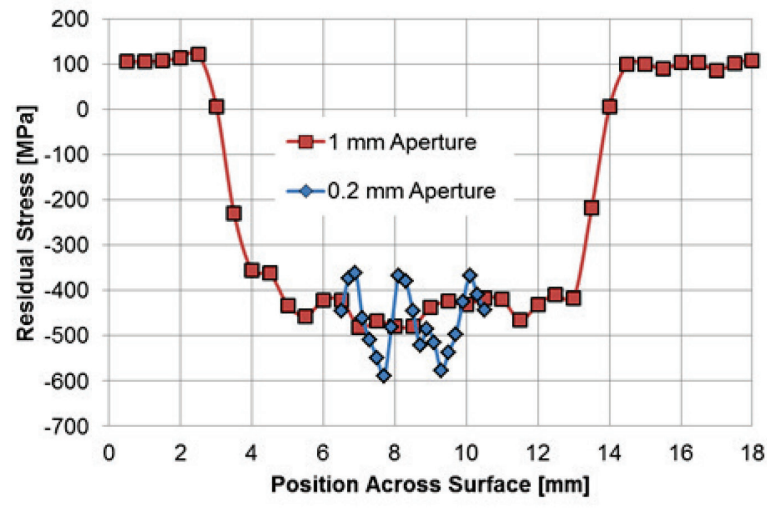

Fig. 5: Residual stress comparison from XRD measurements using two different aperture selections (1 tape layer).
Due to the observed variation of surface residual stress, further XRD measurements were performed with a $0.2 \mathrm{~mm}$ aperture as shown in Fig. 5. The averaging effect of the 1 $\mathrm{mm}$ aperture is significant for a $2 \mathrm{~mm}$ spot size. The $0.2 \mathrm{~mm}$ aperture highlights the variation in the surface stresses, which is periodic and correlates with the spot pattern. The pk-pk variation was approximately 220 $\mathrm{MPa}$ for a sample treated with $5 \mathrm{GW} / \mathrm{cm}^{2}$ and one tape layer.

Fig. 6 shows the effect of two applications of the LSP process, illustrating that the second layer increases residual stress both at the surface and with depth. In addition the variation across the profile is improved significantly. In industrial applications a third layer is often used, but the previous work showed that for this material the third layer has limited benefit. The point at which the residual stress profile crosses zero improves from approximately $0.9 \mathrm{~mm}$ 


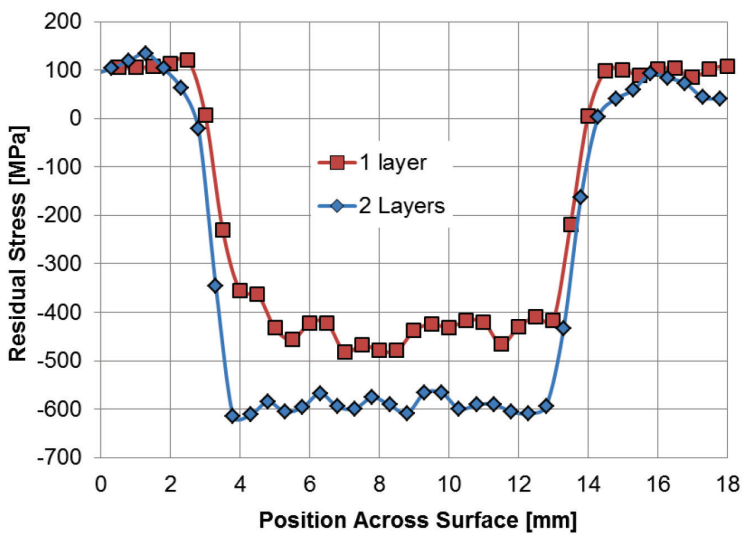

XRD surface residual stress line scans

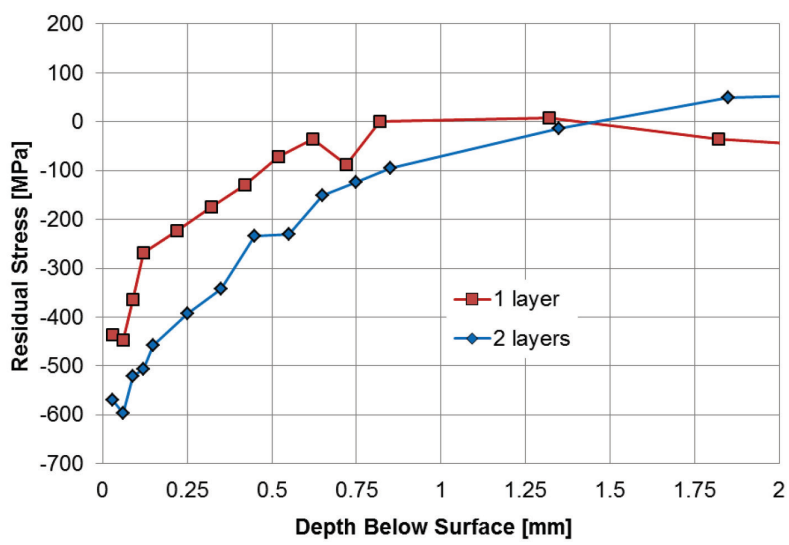

SXRD residual stress depth profiles

Fig. 6: Effect of 1 or 2 tape layers on the induced residual stress values.

to $1.5 \mathrm{~mm}$. Error bars are not shown for clarity of the plots, but typically the strain error was less than $70 \mu \mathrm{m} / \mathrm{m}$.

Fig. 7 shows SXRD data for the LSPwC process with the surface stress value take from the XRD measurements. The samples had a rougher surface than LSP as shown in Fig. 9. The process results in a thin oxide layer on the surface and a recast layer below that. The effect of this on the SXRD data is that the $\mathrm{d}_{\text {zero }}$ in the first $100 \mu \mathrm{m}$ is different from the bulk and this affects the data over the first $150 \mu \mathrm{m}$, due to the beam width. The plots in Fig. 7 are thus a combination of the two measurement processes and the focus is on the profile after $250 \mu \mathrm{m}$. The benefit of increased coverage in the LSPwC process is shown by the improved depth and amplitude of residual stress when $\mathrm{Np}$ increases from 16.85 to 33.75. Decreasing spot size has a benefit on the surface residual stress but the depth profile improves with the increase in spot size from 0.6 to $0.8 \mathrm{~mm}$.

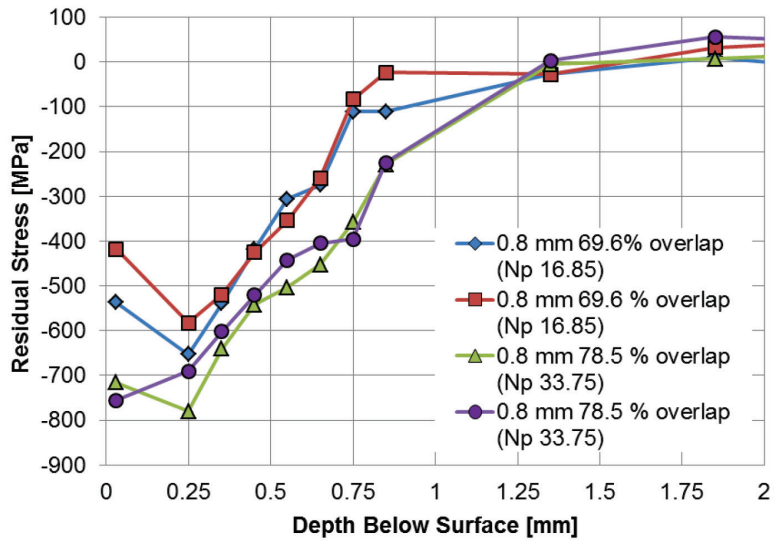

LSPwC samples -SXRD for overlap

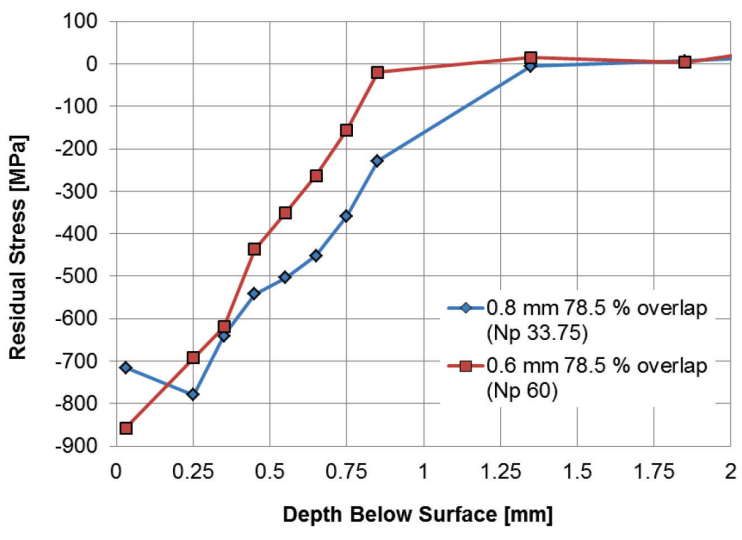

LSPwC samples -SXRD data for spot size

Fig. 7: Coverage and spot size effect. 
LSP and LSPwC both show a significant improvement over SP with the depth of residual stress at the zero crossing point increasing from 0.25 to $1.5 \mathrm{~mm}$. LSPwC has a better depth profile than LSP, but the process is slower due to the smaller spot size and the higher overlap. In industrial applications the time required to treat a component will be one of the deciding parameters.

The benefit of improved surface roughness is clearly illustrated in Fig. 9, using the SP at $200 \%$ coverage condition as a reference, the surface roughness $\mathrm{Ra}$ improves by a factor of 3.24 for LSPwC and 12.0 for LSP. This is particularly beneficial in environments where stress corrosion cracking can take place. The improved surface roughness will also aid in assembly of turbine blades into the location slots on the turbine rotor.

\section{Conclusions}

The LSP and LSPwC treatment proceses are both producing the desired results on $12 \mathrm{Cr}$ steel flat samples, with significantly improved residual stress

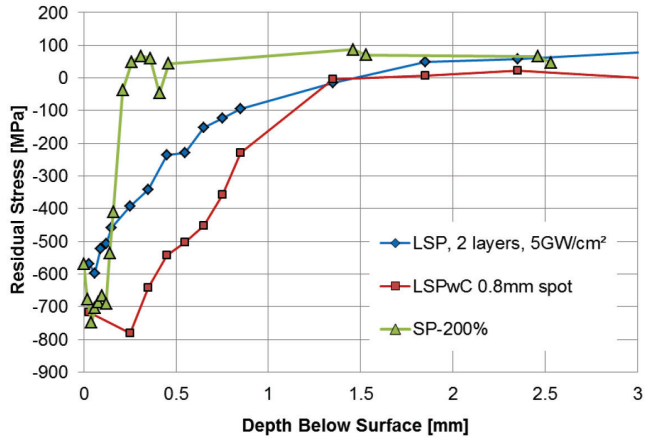

Fig. 8: Comparison of techniques

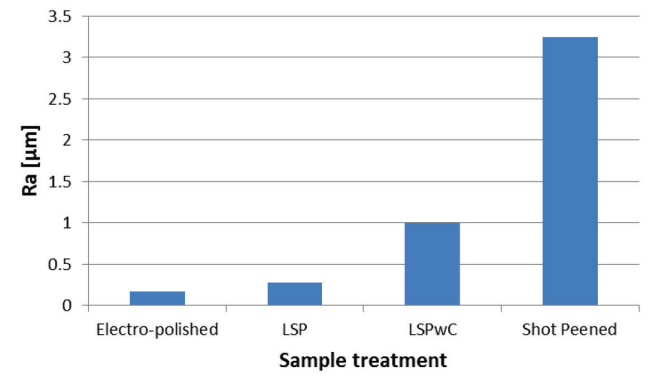

Fig. 9: Surface roughness. profiles when compared to SP.

Residual stresses induced from LSP (2 mm spot, $21.4 \%$ overlap, $5 \mathrm{GW} / \mathrm{cm}^{2}$ and one tape layer) vary periodically by approximately $\pm 220 \mathrm{MPa}$ across the treated area.

The depth profile on a sample improves after two layers with treatment and the surface stress shows less variation. This is important when considering the time required for application on industrial components.

The residual stress transition from the treated to un-treated regions was very smooth and did not exhibit a sharp tensile peak.

\section{Acknowledgements}

The European Synchrotron Radiation Facility (ESRF) for beam time allocation, and assistance from Dr. Thomas Buslaps in experiment ME-1440.

\section{References}

[1] M. Newby, M. N. James and D. G. Hattingh, Finite element modelling of residual stresses in shot-peened steam turbine blades, Fatigue Fract. Eng. Mater. Struct. 37(7) (2014) 707-716. https://doi.org/10.1111/ffe.12165

[2] M. Newby, D. Glaser, and C. Polese, Laser Shock Peening Process Development for Turbine Blade Refurbishment Applications Using a Commercial 'Mid-Range' Energy Laser, in 6th International Conference on Laser Peening and Related Phenomena, 2016.

[3] K. R. Kuveya, C. Polese and M. Newby, Laser Peening versus Shot Peening Effects on Residual Stress and Surface Modification of X12CrNiMo12 Turbine Blade, in 6th International Conference on Laser Peening and Related Phenomena, 2016. 